\title{
DOOR RECOGNITION IN CLUTTERED BUILDING INTERIORS USING IMAGERY AND LIDAR DATA
}

\author{
L. Díaz-Vilariño a, *, J. Martínez-Sánchez ${ }^{\mathrm{a}}$, S. Lagüela ${ }^{\mathrm{a}}$, J. Armesto ${ }^{\mathrm{a}}$, K. Khoshelham ${ }^{\mathrm{b}}$
}

\author{
a Applied Geotechnologies Research Group, University of Vigo, ETSE Minas, 36310 Vigo (Spain)- (lucia, \\ joaquin.martinez, susiminas, julia)@uvigo.es \\ ${ }^{b}$ Faculty of Geo-Information Science and Earth Observation, University of Twente, P.O. Box 217, Enschede 7514 AE, \\ The Netherlands (k.khoshelham)@utwente.nl
}

\section{Commission V}

KEY WORDS: 3D modelling, feature extraction, imagery, terrestrial laser scanning.

\begin{abstract}
:
Building indoors reconstruction is an active research topic due to the importance of the wide range of applications to which they can be subjected, from architecture and furniture design, to movies and video games editing, or even crime scene investigation. Among the constructive elements defining the inside of a building, doors are important entities in applications like routing and navigation, and their automated recognition is advantageous e.g. in case of large multi-storey buildings with many office rooms. The inherent complexity of the automation of the recognition process is increased by the presence of clutter and occlusions, difficult to avoid in indoor scenes. In this work, we present a pipeline of techniques used for the reconstruction and interpretation of building interiors using information acquired in the form of point clouds and images. The methodology goes in depth with door detection and labelling as either opened, closed or furniture (false positive).
\end{abstract}

\section{INTRODUCTION}

In the last decade, 3D building reconstruction has been a research of interest due to the increasing demand of realistic and accurate building models, which are requested as an input source for a variety of purposes.

The representation of building interiors can support a wide range of applications in many fields, from architectural planning, to lighting analysis, crime scene investigation or indoor navigation. Specifically, openings of the building (windows and door) are of primary interest due to their unchanging nature within the building, especially when compared to furniture, as well as their influence of natural illumination and emergency route planning, among others. The geometric detail and the semantic content of existing indoor models strongly depend on the application area for which models are created. In this way, Building Information Modelling (BIM) provide highly detailed 3D indoor models that support a large amount of semantic data; while the representation of building interiors in GIS is limited in both geometry and semantic contents. This is due to the fact that, 3D GIS models are aimed at urban, global and large-scale purposes.

Manual generation of a building model is a time-consuming process that requires expert knowledge (Tang, et al, 2010; Gonzalez-Aguilera et al, 2012). Reconstruction tools based on the interpretation of measured data such as LiDAR Data and Images are frequently used for the automatic interpretation and reconstruction of building geometry.

One of the key challenges to the automation of the reconstruction of building interiors is the presence of clutter and occlusions, caused by furniture and other objects. To be useful, modelling algorithms should be functional in unmodified environments since it is not practical to remove the furniture and objects of an indoor scene prior data acquisition. Their specific challenges are, on the one hand, occluding objects that can block the visibility of the structural building surfaces (i.e. walls, ceilings or floors) causing absence of measured data; on the other hand, they can be erroneously interpreted as parts of the model itself. For example, a large bookshelf or cupboard can be confused with a door because their size and shape can be similar; or the frame of a picture can also be not clearly distinguishable from a window.

Despite the presence of clutter and occlusions in building interiors, some approaches have dealt successfully with the reconstruction of structural elements of indoor scenes from imagery and/or point cloud data. Some methods based on datadriven approaches are presented by (Valero et al, 2012; DíazVilariño et al 2014) and prior knowledge is used by (Budroni and Boehm, 2010; Becker et al, 2003).

Regarding openings, as windows can be modelled also from outside, most literature about windows reconstruction is focused on facades because they present lower occlusion levels and repetitive patterns. Nevertheless, several methods have been developed to extract windows from indoor environments. Adán et al, (2011) and Previtali et al. (2014) detect openings in indoor scenes by analysing data density and classifying lowdensity areas as openings, thus limiting the scope to low-density windows and doorways. Demisse et al, (2013) use thermally coloured point clouds for detecting windows and labelling them as closed or open according to the temperature difference between walls and windows.

\footnotetext{
* Corresponding author.
} 
In contrast to windows, there is not much literature on door reconstruction. From point clouds, if the doors are open, they can be easily detected as a hole in the wall. If they are closed, detection becomes more difficult because their descriptive features are highly dependent on the quality of input data. From imagery, similar size and shape objects can be erroneously understood as doors.

In this work, we propose a simple but effective methodology based on automatic data-driven approach for the reconstruction of building indoor scenes using both 3D point clouds and RGB images, going in depth with door detection and classification. The methodology is tested through a case study, acquired under unmodified-furniture conditions.

Specifically, an image-based algorithm using the Generalized Hough Transform (GHT) is developed for the detection of door candidates in orthoimages. Then, the point cloud is used as a ground-truth for the distinction of doors from other objects with similar size and shape and the classification of all of them into three categories: open doors, closed doors and furniture.

\section{METHODOLOGY}

The approach developed for modelling building interiors allows the automatic reconstruction of envelope and doors, starting from point clouds and imagery.

The workflow of the proposed methodology is shown in figure 1. The initial step (section 2.2.) involves the segmentation of the point cloud and the recognition and reconstruction of the building elements that compose the indoor-scene envelope (walls, floor and ceiling). The walls obtained are used as the basis for the generation of orthoimages (section 2.3.) which will be the input data for the following step, the detection of door candidates (section 2.4). The final step consists of the classification and pruning of door candidates (section 2.5.).

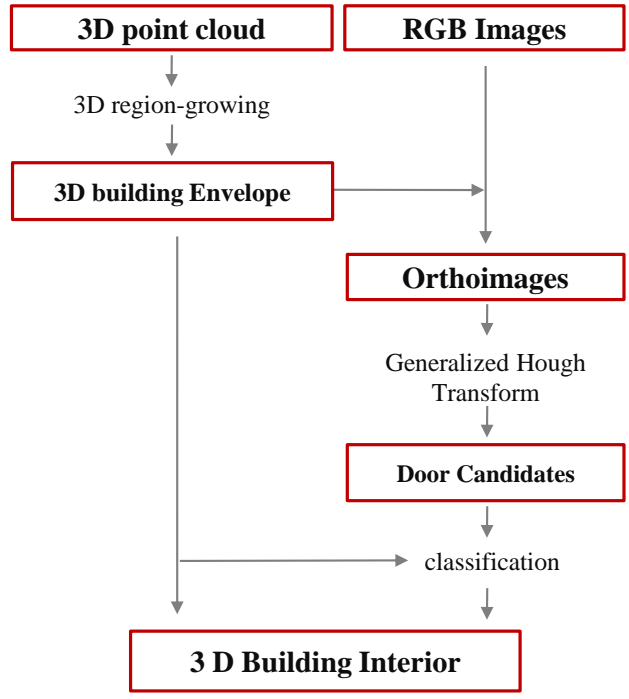

Figure 1. Workflow of the proposed methodology.

\subsection{Data acquisition}

The data set includes point clouds and images obtained from a single hybrid acquisition system formed by a Terrestrial Laser Scanner (TLS), model Riegl LMS Z-390i, and a high-resolution camera firmly mounted on its top (Nikon D200 with a $20 \mathrm{~mm}$ lens).

On the one hand, the Internal Calibration Parameters of the camera are calculated following the photogrammetric calibration process of self-calibration bundle adjustment based on flat check pattern images (Brown, 1971). On the other hand, the External Orientation Parameters (EOPs) are obtained through a point matching process using reflective targets. Therefore, the geometric relation between each laser point and the camera is known.

The laser scanner presents a field of view of $360^{\circ}$ horizontally and $80^{\circ}$ vertically, which implies missing information from the immediate ceiling and floor on top and under it. The choice of number of scanner positions and their location is made by the user depending on the shape complexity of the indoor scene, trying to maximize the area of interest acquirable from each of them.

\subsection{Envelope segmentation and reconstruction}

The acquired point cloud is rotated for its alignment with the main building axis and submitted to a curvature study based on the Principal Component Analysis (PCA) by using the covariance method (Jolliffe, 2002). PCA involves the computation of the eigenvalues $\left(\lambda_{\mathrm{i}}\right)$ and eigenvectors $\left(\mathrm{e}_{\mathrm{i}}\right)$ of the covariance matrix $(\mathrm{COV})$ of a neighbourhood $(\mathrm{k})$ of each point (i). The points, part of these neighbourhoods, are searched according to the minimum Euclidean distance by applying a $\mathrm{K}$ Nearest Neighbour $(\mathrm{KNN})$ procedure.

$$
\begin{gathered}
\operatorname{COV}(X)=\left[\begin{array}{ccc}
\sigma_{x}^{2} & \sigma_{x y} & \sigma_{x z} \\
\sigma_{x y} & \sigma_{y}^{2} & \sigma_{y z} \\
\sigma_{x z} & \sigma_{y z} & \sigma_{z}^{2}
\end{array}\right] \\
\sigma_{X}^{2}=E\left(x^{2}\right)-E(x)^{2}=\frac{1}{k} \sum_{i=1}^{k}\left(x_{i}-\bar{x}\right)^{2} \\
\sigma_{x y}=E(x y)-E(x) E(y)=\frac{1}{k} \sum_{i=1}^{k}\left(x_{i}-\bar{x}\right)\left(y_{i}-\bar{y}\right)^{2}
\end{gathered}
$$

Where, $\mathrm{E}(\mathrm{x})$ is the expected value for an axis $(E(x)=\bar{x})$, and $\sigma_{x}^{2}$ and $\sigma_{x y}$ denote the variance and covariance values, respectively.

Eigenvalues are used afterwards to derive a curvature feature, which can act as an indicator of the planarity of each point neighbourhood: the eigenvector associated to the smallest eigenvalue is considered as the normal vector to the surface of each point, since it is the direction vector of the plane with the lowest probability of being the fitting plane. Therefore, if the point was on a planar surface, the variance should be nominally zero.

Then a normal vector smoothing is performed by averaging each point normal vector with the normal vectors of the points in its neighbourhood, $\mathrm{k}$.

$$
n_{i}^{\prime}=n_{i}+\sum_{i \neq j}^{k} w_{i j} n_{j}
$$

The averaging is carried out with a weighting factor $w_{i j}$ which depends on the distance between points $d_{i j}$, where, $\mathrm{n}_{\mathrm{i}}^{\prime}$ denotes the averaged normal of point $i$. 


$$
w_{i j}=\frac{\frac{1}{d_{i j}}}{\sum_{k, i \neq j} \frac{1}{d_{i j}}}
$$

Then, a seeded region-growing algorithm (Rabbani et al, 2006) is developed for the detection of planar surfaces. For each iteration of the algorithm, the point with lowest curvature value is chosen as a region seed candidate. Following, a regiongrowing step in the algorithm includes in the region all those points satisfying the following conditions:

1. The difference between the direction of its normal vector (i.e. the eigenvector associated to the smallest eigenvalue $\lambda_{0}$ ) and the region normal vector is below a certain threshold $\left(\alpha_{t h}\right)$.

2. The distance between the point and the planar region is below a certain threshold $\left(D_{t h}\right)$.

The algorithm is computed until all points are assigned to a region, even though only regions with the highest number of points are used in successive steps.

After segmentation, horizontal regions are automatically classified into "ceiling" and "floor" according to the $\mathrm{Z}$ component of their coordinates, while vertical regions are submitted to a visual inspection for their identification and labelling into: "walls" or "furniture".

Finally, RANSAC (Fischler and Bolles, 1981) is applied for achieving the best fitting plane to each region and the 3D building envelope is reconstructed by intersecting these planes assigned to walls, floor and ceiling in order to obtain the boundary points that define each element.

\subsection{Orthoimages generation}

Assuming that building interiors are composed of vertical walls with rectangular shapes, the 3D boundary points that define each wall are used to determine the $2 \mathrm{D}$ coordinate system of the orthoimage plane of each wall. The origin of the coordinate system is established in the lower-left boundary point from an interior-scene point of view.

According to the theory of optical projection, the rays corresponding to each object can intersect on the projective plane by adjusting the External Orientation Parameters of each image. Therefore, after defining the orthoimage plane and the resolution of the image to be generated, object $3 \mathrm{D}$ points are transformed from the laser scanner coordinate system to the camera coordinate system through an exterior rotation $(\mathrm{R})$ and translation $(\mathrm{T})$.

$$
\left(\begin{array}{l}
x \\
y \\
Z
\end{array}\right)=R *\left(\begin{array}{l}
X-T_{x} \\
Y-T_{y} \\
Z-T_{z}
\end{array}\right)
$$

Both rotation and translation matrices are obtained from the geometric relation between the laser scanner and the camera, given by the acquisition device, and the rotation matrix obtained from the point cloud alignment with the main building axis.

Then, resulting points are projected to the image plane and RGB values are obtained through the perspective projection of the $3 \mathrm{D}$ points using a pinhole camera model,

$$
x^{\prime}=f \cdot \frac{x}{z}
$$

$$
y^{\prime}=f \cdot \frac{y}{z}
$$

where $(\mathrm{x}, \mathrm{y}, \mathrm{z})$ represent a point projected onto the image plane, $f$ is the focal length of the camera and $\left(x^{\prime}, y^{\prime}\right)$ refer to the coordinates of the image pixel.

Lens distortion is calculated and corrected to make possible the correction of the difference between the actual camera projection and the camera model, which is introduced by the lens. The Interior Orientation Parameters consist of three radial distortion coefficients $\left(\mathrm{K}_{1}, \mathrm{~K}_{2}, \mathrm{~K}_{3}\right)$ and two decentring distortion coefficients $\left(\mathrm{P}_{1}, \mathrm{P}_{2}\right)$ are respectively applied to the computation of the radial $\left(d_{r a d}\right)$ and decentring $\left(\mathrm{d}_{\mathrm{xdec}}, \mathrm{d}_{\mathrm{ydec}}\right)$ corrections, which are undone to obtain the distorted pixel coordinates.

$$
\begin{gathered}
d_{\text {rad }}=K_{1} r^{2}+K_{2} r^{4}+K_{3} r^{6} \\
d_{x d e c}=P_{1}\left(2 x^{\prime 2}+r^{2}\right)+P_{2} 2 x^{\prime} y^{\prime} \\
d_{y d e c}=P_{2}\left(2 y^{\prime 2}+r^{2}\right)+P_{1} 2 x^{\prime} y^{\prime}
\end{gathered}
$$

Where, $r=\sqrt{x^{\prime 2}+y^{\prime 2}}$.

As the image acquisition is carried out for the complete horizontal angle of view $\left(0^{\circ}-360^{\circ}\right)$, the azimuth (regarding the Camera Coordinate System) of the ray formed between each pixel and the camera projection centre is calculated and compared with the orientation of each image. If the azimuth of the ray is within the field of view of the image, the RGB value is adopted. If this condition is not verified, the point is not in the image frame.

Finally, as the image acquisition is performed with $10 \%$ overlap between consecutive images, a linear transition (Lui et al, 2009) is implemented in order to smooth transition areas and eliminate edge seams caused by a direct average fusing.

\subsection{GHT for doors detection}

The Generalized Hough Transform (GHT) (Ballard, 1981) is applied for detecting door candidates in the orthoimages previously generated.

In the original Hough Transform (Hough, 1962), a straight line is represented by the Hesse form, where $\rho$ is the distance from the application point of the normal vector to the origin of $X Y$ image space and $\theta$ is its angle with the $\mathrm{X}$-axis.

$$
\rho=X \cos \theta+Y \sin \theta
$$

The method is based on the transformation of the image space $(\mathrm{XY})$ into a $2 \mathrm{D}$ parameter space $(\rho \theta)$. Each pixel in the image corresponds to a curve representing all the parameters of the lines of a bundle of straight lines that have this pixel as intersection point. Therefore, the objective of this line detection process is to find this intersection point. The parameter space is discretized through an accumulator array consisting of bins where votes from edge pixels in the image space are stored. The bin that receives the maximum number of votes determines the solution.

While the Hough Transform is used to detect objects defined with few parameters such as lines or planes, the GHT transforms the shape detection problem into a maximum analysis problem, so that arbitrary shapes can be detected, even 
being robust to partial occlusion and clutter (Khoshelham, 2007).

The algorithm uses edge information to define a mapping from the orientation of an edge point to a reference point of the shape. Doors can be assumed as rectangles with vertical orientation. For the performance of edge detection, true colour orthoimages are converted to grayscale images, where edges are found by the Canny operator. Then, the resulting edges are submitted to an orientation filter through which $90^{\circ}$ and $180^{\circ}$ oriented edge pixels are selected for computation.

The shape of the door (rectangle), defined by length ( $r$ ) and direction $(\beta)$, is previously stored in a table, called R-Table, where the gradient direction $(\varphi)$ serves as an index.

The process starts by selecting an arbitrary reference point for the door $\left(\mathrm{X}_{\mathrm{C}}, \mathrm{Y}_{\mathrm{C}}\right)$. For every resulting edge pixel on the shape edge, the gradient direction $(\varphi)$ as well as the length (r) and the direction $(\beta)$ of a vector connecting the boundary pixel to the reference point are computed. For each couple $(r, \beta)$ found in the R-Table, the accumulator array bin is increased in one vote. At the end of this voting process, the bin with the maximum number of votes indicates the reference point and the edge pixels of the most probable candidate. In order to refine the results, neighbour candidates are supressed.

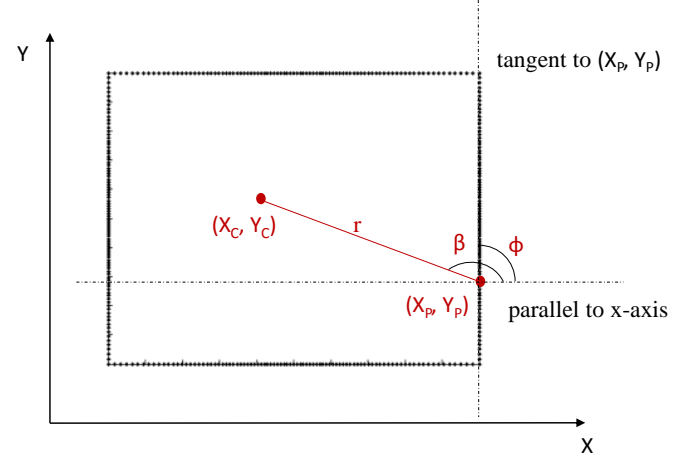

Figure 2. Parameters involved in the GHT for door-candidate detection.

As doors are assumed as rectangles with vertical orientation, four parameters are considered for their definition: two coordinates of the centre, width and height. They are used as constraints to enforce the detection of door candidates.

\subsection{Candidate classification and pruning}

Due to the detection process, objects with size and shape similar to doors can be detected as false positives. In order to reduce the false positive rate, a candidate pruning method based on the analysis of the segmented planar regions and the original 3D point cloud is implemented.

After segmentation, points on closed doors can be labelled as wall points depending on the distance thresholds in the region growing $\left(D_{t h}\right)$ and the RANSAC $\left(D_{R}\right)$ steps. Lowering these thresholds can be a solution for contiguous planes, but it could result in over-segmentation of large walls. Therefore, enhancing the pruning method with classification capabilities results in a more robust methodology.

In a first step, points that are projected orthogonally onto the rectangular candidate are taken into account. The points that are at higher distance than the threshold distance from the wall plane are discarded. This threshold distance is coincident with the candidate width, in order to consider enough space to open and pass through a door opening.

Following, a histogram of the point-to-plane distance is computed and inspected. Theoretically, a zero-valued histogram describes an open door, whereas closed doors are modelled with a Dirac delta function. The same function describes an office cupboard, but in this case, the delta appears displaced to a position coincident with the cupboard depth with respect to its contiguous wall. In figure 3 , a graphical drawing of this classification is shown. For real-world histograms, similar functions are expected, but taking into account the precision of the TLS, the computation of the candidate boundaries, and the as-built condition of the building. The classification of the candidate is achieved looking for maxima in the histogram and comparing the maxima value with the mean value of the histogram. Maxima points that are three times higher than the mean value are detected as peak values in the histogram.
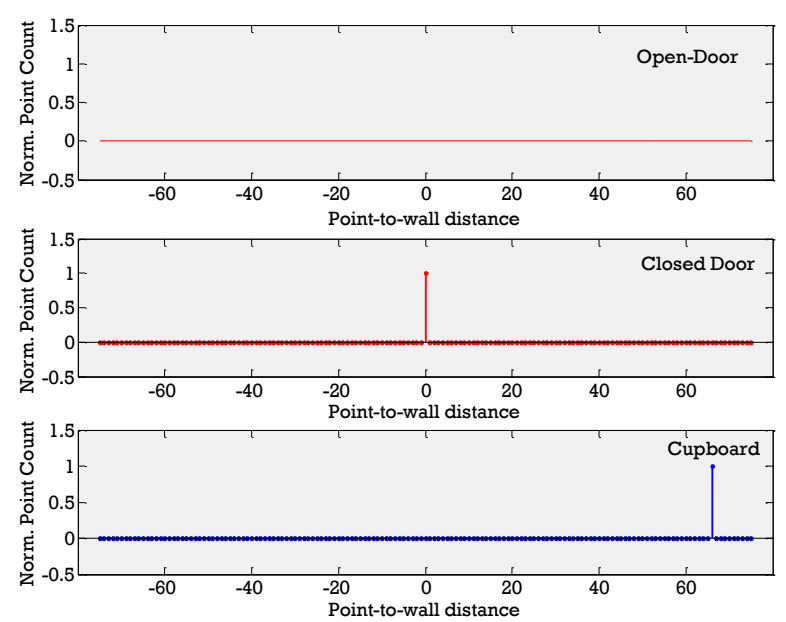

Figure 3. Theoretical normalized density of points. For opendoors, there are not any points in front or behind the wall. For closed-doors and cupboards, all points are on the plane, near to the wall for doors and away from it for cupboards.

To summarize, depending on the number of peaks in the histogram and their values, candidates are classified as follows; Candidates with constant histograms are labelled as "open doors". Candidates with a single peak value are labelled as "closed door" or "office cupboard" depending on the position of the maximum. Finally, candidates with a histogram with two or more peaks are labelled as "non-door" objects.

\section{RESULTS AND DISCUSSION}

The methodology is tested with a case study: an indoor scene in an academic building. Data is acquired from one Scan Position placed in the centre of the scene, under two different contexts: closed and open doors respectively.

The geometric acquisition is carried out with an angular resolution of $0.08^{\circ}$ and point clouds are submitted to a filtering process performed by using an octree filter (size of the cube equal to $0.02 \mathrm{~m}$ ). Regarding images, the complete scene is captured with 10 images, with a $10 \%$ overlap between consecutive images. The device used (section 2.1) presents a field of view of $360^{\circ}$ horizontally and $80^{\circ}$ vertically, which implies missing information from the immediate ceiling and 
floor on top and under it. However, this lack of data does not affect the next steps in the proposed workflow.

With regard to segmentation, a curvature analysis is performed by analysing each point supported by a neighbourhood of its 50 closest points (Díaz-Vilariño et al, 2013). The region-growing step is carried out taking into account, on one side, a high angular threshold $\left(\alpha_{t h}\right)$ of $75^{\circ}$ and on the other side, a small connectivity threshold $\left(D_{t h}\right)$ of $0,05 \mathrm{~m}$. As this step aims to segment just envelope elements such as walls, ceilings and floors, these parameters are chosen to avoid over-segmentation. In this way, breakings of smooth areas are ensured to appear only on the edges between consecutive elements. Finally, as building interiors are usually highly cluttered and the point cloud is processed without cleaning pre-processing, small size regions (minimum size of 350 points) are rejected for further processing.

As a result of the segmentation procedure, 36 planar regions are detected (figure 4). They are submitted to a visual recognition process, and 7 planar regions are classified as "walls", 1 as "floor" and 1 as "ceiling", while remaining regions are labelled as "furniture".

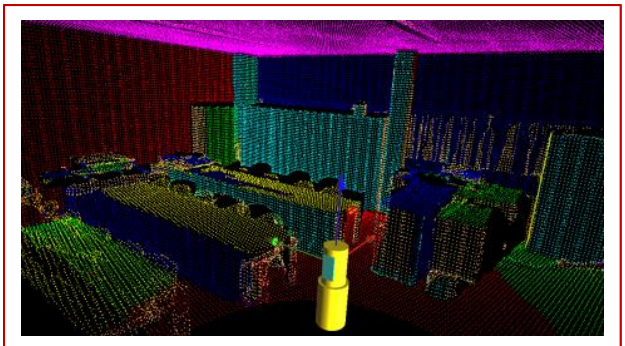

Figure 4. The segmented planar regions are shown in different colours.

After the geometric segmentation and classification, RANSAC is applied with a $0.015 \mathrm{~m}$ distance threshold $\left(D_{R}\right)$ for achieving the best fitting plane of those regions classified as envelope components, which are used for the $3 \mathrm{D}$ building reconstruction through their intersection.

The four 3D boundary points that define each wall are used for orthoimage generation in both contexts (closed and open door), considering a $2 \mathrm{~cm}$ resolution. Figure 5 shows the $3 \mathrm{D}$ building envelope texturized with the orthoimages in the closed-door context.

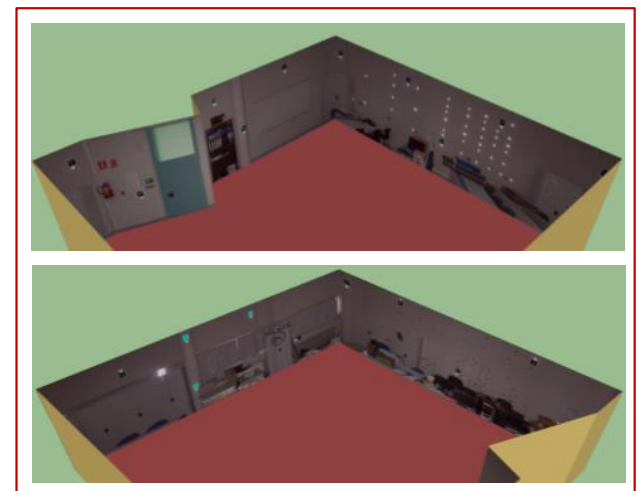

Figure 5: The 3D building interior is textured with the orthoimages.

Orthoimages are submitted to the door detection approach based on the GHT. All of them are processed together, so that the parameters used are common for all the orthoimages of the case of study.

The 4D accumulator is constructed taking into account as initial parameters a minimum and maximum door width $(0.8 \mathrm{~m}$ and $1.2 \mathrm{~m}$, respectively) and a minimum and maximum door height $(1.8 \mathrm{~m}$ and $2.4 \mathrm{~m})$.

As the case of study is an academic building interior, the presence of furniture with similar shape and size as doors is frequent. Given that the number of doors in each wall is not known, 25 bins are searched in each orthoimage, resulting in an over-detection of door candidates. Moreover, neighbours of selected bin are supressed within a $30 \mathrm{~cm}$ threshold.

Finally, a final candidate selection is carried out for all the bins. First, all the orthoimages are considered together, and the bins with a voting rate inferior to the global $95^{\text {th }}$ percentile are deselected. Next, each image is considered individually, and the most voted candidates are selected by rejecting the values below the individual $95^{\text {th }}$ percentile. In this way, the results of this step are the most voted door candidates for each wall, considering the whole indoor building.

In this door detection approach, the door (in both open and closed context) and 3 bookshelves are detected as door candidates due to their similar size and shape (figure 6).

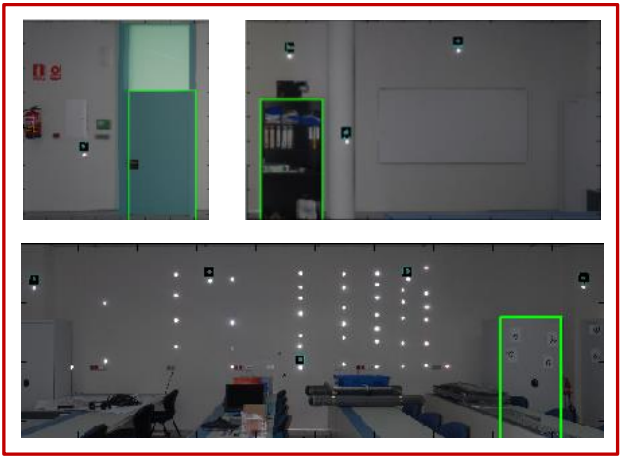

Figure 6. A closed-door (upper-left), a bookshelf (upper-right) and a cupboard (down) are detected as door candidates.

Next, candidate classification is applied, rejecting falsepositives and labelling doors if applicable. Figures 7 to 10 describe the different cases analysed in the case study, the three first figures belong to the first context where the door was open, whereas the last figure belongs to the second context with closed-door. In this second context, the same candidates were detected as non-door objects.

In figure 7 an open door is classified because there is no peak value in the histogram.

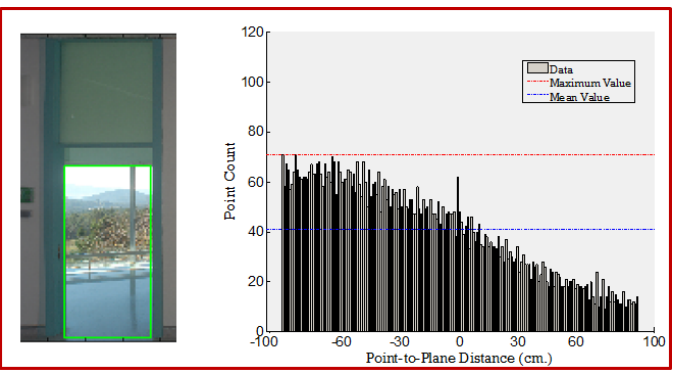

Figure 7. Histogram of point-to-plane distances: Open door case. 
A single peak value is detected $56.5 \mathrm{~cm}$ away from the wall plane in figure 8 , resulting in the classification of an office cupboard. The depth of the cupboard is $43 \mathrm{~cm}$. but it is displaced from the wall a total distance of $55.2 \mathrm{~cm}$.
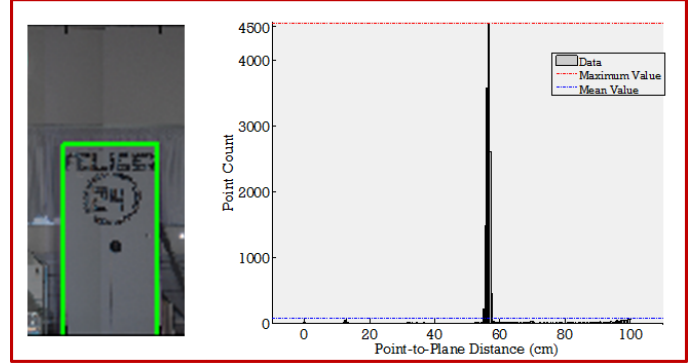

Figure 8. Histogram of point-to-plane distances: cupboard case.

Three peak values are detected in the histogram of figure 9 at 2 $\mathrm{cm} ., 32 \mathrm{~cm}$. and $34 \mathrm{~cm}$. from the plane resulting in the classification of a non-door object. This object corresponds to a bookshelf that has a total depth of $33.5 \mathrm{~cm}$. whereas the shelves have a depth of $32 \mathrm{~cm}$. The peak value at $2 \mathrm{~cm}$ corresponds to the inner surface of the bookshelf.
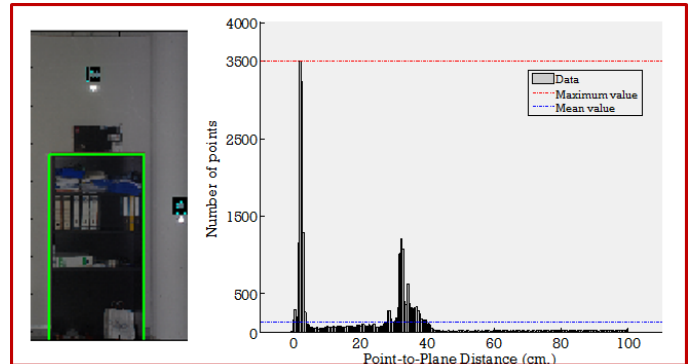

Figure 9. Histogram of point-to-plane distances: a bookshelf is detected as false-positive door and labeled as NON-DOOR object.

Finally, figure 10 shows the histogram of a closed door. The only peak value is positioned at $-5 \mathrm{~cm}$ from the wall plane, because the door is aligned to the outer face of the wall. In this picture, some points of those closer to the wall are distinguishable. These points correspond to the doorjambs and lintel.
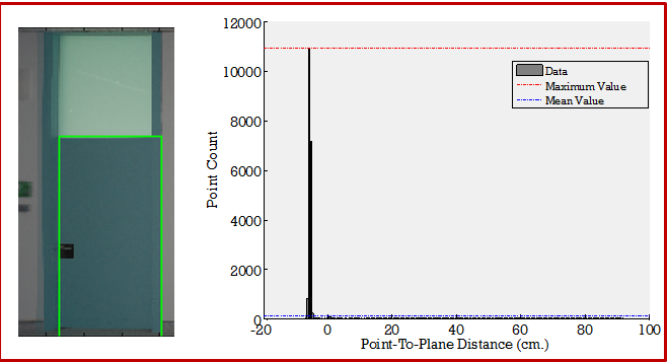

Figure 10: Histogram of point-to-plane distances: closed door segmentation in the second context.

As a result of this step, three false positive candidates were easily checked and removed and one true positive was verified as a door. Moreover, false negatives were not obtained.

Finally, the boundary points of the detected doors are projected onto the correspondent wall planes for the reconstruction of the complete 3D building interior (Figure 11).

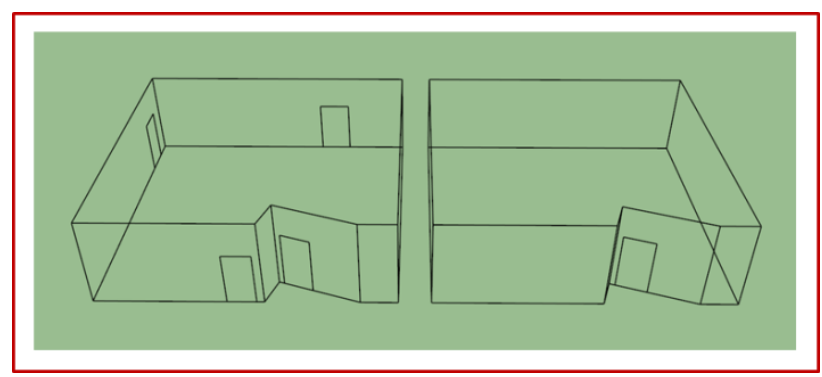

Figure 11. Building indoor model before (left) and after doorcandidate pruning (right).

\section{CONCLUSIONS}

In this paper, we present a pipeline of techniques used for the reconstruction and interpretation of building interiors. While the point cloud is the basis for modelling the envelope of the scene, door candidates are detected in the orthoimages. The point cloud is also used for the distinction of doors from other objects with similar size and shape, and for the classification of doors as closed or open. The proposed methodology optimizes the result of the 3D model by maximizing the information acquired without increasing acquisition time, given that both the point cloud and the corresponding images are captured at the same time. What is more, the combined use of images and point clouds increases the capabilities for door detection, making possible its distinction from similar furniture such as bookshelves, as well as their detection with independence of their condition of closed or open door.

As the input of the GHT are grey level images of edges, the door candidates detection is intensity-dependent and its accuracy can be influenced by lightness effect.

Future work will deal with the assessment and improvement of the methods presented here. First, this includes a thorough evaluation of the performance in different cases of study, towards error analysis. Second, the aim is at extending this methodology to windows.

\section{Acknowledgements}

Authors would like to give thanks to the Government of Spain for the financial support given through human resources grants (FPU AP2009-1144, FPU AP2010-2969).

\section{REFERENCES}

Adán, A., Xiong, X., Akinci, B., Huber, D., 2011, Automatic creation of semantically rich $3 \mathrm{~d}$ building models from laser scanner data, Proceedings of the International Symposium on Automation and Robotics in Construction (ISARC).

Ballard, D.H., 1981. Generalizing the Hough transform to detect arbitrary shapes, Pattern Recognition, 13(2), pp. 111-122.

Becker, S., Peter, M., Fritsch, D., Philipp, D., Baier, P. \& Dibak, C. 2013, Combined Grammar for the Modeling of Building Interiors, ISPRS Annals of the Photogrammetry, Remote Sensing and Spatial Information Sciences, II-4/W1, pp 1-6, 2013.

Brown DC, 1971, Close-range camera calibration, Photogrammetric engineering, 37, (8), 855-866. 
Budroni, A., Boehm, J., 2010. Automated 3D reconstruction of interiors from point clouds. International Journal of Architectural Computing 8(1), 55-73

Demisse, G., Borrmann, D., and Nüchter, A., 2013, Interpreting Thermal 3D Models of Indoor Environments for Energy Efficiency. Proceedings of the 16th IEEE International Conference on Advanced Robotics (ICAR '13)

Díaz-Vilariño, L., Lagüela, S., Armesto, J., Arias, P., 2013, Semantic as-built $3 \mathrm{~d}$ models including shades for the evaluation of solar influence on buildings, Solar Energy, 92, 269-279

Díaz-Vilariño, L., Lagüela, S., Armesto, J., Arias, P., 2014, Indoor daylight simulation performed on automatically generated as-built 3D models, Energy and Buildings, 68, 54-62

Fischler, M.A., and Bolles, R.C., 1981. Random sample consensus: a paradigm for model fitting with applications to image analysis and automated cartography, CACM 24 (6), 381395 .

Jolliffe, I.T., 2002, Principal Component Analysis, second ed., Springer, New York.

Gonzalez-Aguilera, D., Del Pozo, S., Lopez, G., RodriguezGonzalvez, P., 2012, From point cloud to CAD models: Laser and optics geotechnology for the design of electrical substations, Optics \& Laser Technology 44 (5), 1384-1392
Khoshelham, K., 2007, Extending generalized Hough transform to detect 3D objects in laser range data, ISPRS Workshop on Laser Scanning 2007 and SilviLaser 2007, Espoo, Finland, pp. 206-210.

Liu, J. Shen, W. Chen, 2009, Image mosaic technology based on overlapped area linear transition method, 2nd Int. Congress on Image and Signal Processing, Tianjin, pp. 1-3.

Previtali, M., Scaioni, M., Barazzetti, L., and Brumana, R. 2014. Towards automatic indoor reconstruction of cluttered building rooms from point clouds. Int. ISPRS Annals of Photogrammetry, Remote Sensing and Spatial Information Sciences, Vol. II, Riva del Garda, Italy, 8 pages.

Rabbani T, Heuvel FA, Vosselman G. 2006, Segmentation of point clouds using smoothness constraint. IAPRS 36(5):248-53

Tang, P., Huber, D., Akinci, B., Lipman, R., Lytle, A., 2010, Automatic reconstruction of as-built building information models from laser-scanned point clouds: A review of related techniques, Automation in Construction, 19(7), 829-843

Valero, E., Adán, A., Cerrada, C., 2012. Automatic Method for Building Indoor Boundary Models from Dense Point Clouds Collected by Laser Scanners. Sensors 12(12), 16099-16115. 